\title{
The complete genome sequence and analysis of vB_VorS-PVo5, a Vibrio phage infectious to the pathogenic bacterium Vibrio ordalii ATCC-33509
}

\author{
Alex Echeverría-Vega ${ }^{1,2}$, Pablo Morales-Vicencio ${ }^{2}$, Camila Saez-Saavedra ${ }^{2}$, Janja Ceh $^{3}$ and Rubén Araya ${ }^{2,3^{*}}$
}

\begin{abstract}
The bacterium Vibrio ordalii is best known as the causative agent of vibriosis outbreaks in fish and thus recognized for generating serious production losses in aquaculture systems. Here we report for the first time on the isolation and the genome sequencing of phage VB_VorS-PVo5, infectious to Vibrio ordalii ATCC 33509. The features as well as the complete genome sequence and annotation of the Vibrio phage are described; VB_VorS-PVo5 consists of a lineal double stranded DNA totaling $\sim 80.6 \mathrm{~Kb}$ in length. Considering its ability to lyse Vibrio ordalii ATCC 33509, the phage is likely to gain importance in future aquaculture applications by controlling the pathogen and as such replacing antibiotics as the treatment of choice.
\end{abstract}

Keywords: Short genome report, Bacteriophage, Vibrio ordalii, Siphoviridae, DS-DNA virus

\section{Introduction}

The Chilean coast is characterized by the Humboldt Current System, a cold, low-salinity ocean current that is considered one of the most productive marine ecosystems on Earth. Cold, nutrient rich waters are constantly upwelled into the photic zone providing sustenance for primary producers $[1,2]$. This environment offers highly favorable natural conditions for the growth of heterotrophic bacteria [3], e.g., for Vibrio species, known as the main pathogenic bacterial group in Chilean aquaculture $[4,5]$. One of the most frequently isolated marine Vibrio species in the salmon industry is Vibrio ordalii which has been described as highly pathogenic for larvae reared in hatcheries [6-9], and therefore significantly contributes to production losses. In order to prevent diseases and control infections in fish farms, the intensive use of a wide variety of antimicrobials is applied.

\footnotetext{
* Correspondence: ruben.araya@uantof.cl

${ }^{2}$ Laboratorio de Microbiología Costera, Facultad de Ciencias del Mar y Recursos Biológicos, Universidad de Antofagasta, Angamos 601, Antofagasta, Chile

${ }^{3}$ Instituto de Ciencias Naturales Alexander von Humboldt, Facultad de Ciencias del Mar y Recursos Biológicos, Universidad de Antofagasta, Angamos 601, Antofagasta, Chile

Full list of author information is available at the end of the article
}

However, the poor management of such treatments, e.g., the use of antibiotics in discrete doses as a prophylactic therapy $[10,11]$, has caused enormous damage to the environment [12-14]. Moreover, the increasing development of antimicrobial resistance in natural bacterial communities $[10,15,16]$, calls for stricter regulations of antibiotic use [17]. As a consequence, the interest in phage therapy as an alternative control for bacteria in aquaculture systems has recently re-gained momentum [18-21].

In our quest to find a natural control for $V$. ordalii in aquaculture, we focused our research on isolating phages potentially effective against the pathogen, surveying various marine sources, e.g., sea water, sediment and intertidal filter organisms. Amongst others we tested the filter-feeding Perumytilus purpuratus (Lamarck, 1819), an intertidal mussel common to the northern Chilean coast and a promising source organism for phages as it uptakes and concentrates local microbiota in its gut system. We succeeded in isolating and identifying vB_VorS-PVo5 a novel marine phage belonging to the family Siphoviridae that causes lytic infections in the bacterium V. ordalii ATCC 33509 and therefore qualifies as a potent future candidate to control one of the 
most harmful bacteria in the aquaculture industry. The whole genome sequence of the phage was sequenced on an Illumina MiSeq platform and is described here, presenting the first report of an isolated and sequenced phage that infects the marine bacterium Vibrio ordalii.

\section{Organism information \\ Classification and features}

The Vibrio phage vB_VorS-PVo5 belongs to the Siphoviridae, a family of double-stranded DNA viruses in the order Caudovirales, and forms $\sim 2$-mm diameter plaques when infecting $V$. ordalii type strain ATCC 33509.

The phage was isolated from macerated specimens of the mussel Perumytilus purpuratus, collected in the intertidal zone off the Antofagasta coast in Chile $\left(23^{\circ}\right.$ $42^{\prime} 00^{\prime \prime S}$; 70²5'88"W). Transmission electron microscopy of purified phage particles (Fig. 1) revealed an icosahedral capsid ( $~ 85 \mathrm{~nm}$ diameter) and a distinguishable long tail ( 150 nm length). The capsid encapsulates a linear double-stranded DNA genome of a length of $80,578 \mathrm{bp}$. An alignment of the DNA polymerase gene, a method commonly applied as a viral phylogenetic marker [22], demonstrated that vB_VorSPVo5 phage clusters closely to (a) Vibrio phage pVp_1 GB JQ340389, a known predator of $V$. parahaemolyticus ATCC 33844 previously isolated from the Yellow Sea coast in Korea [23], and (b) Vibrio phage phi 3 GB AJF40879, associated with Vibrio cholerae 1051 and previously isolated in Russia (Fig. 2). All sequences were collected from NCBI and aligned using CLUSTALW [24]; the evolutionary analysis was inferred through the neighbor-joining method using MEGA6 [25] under auto settings.

\section{Genome sequencing information Genome project history}

The bacteriophage vB_VorS-PVo5 was selected for genome sequencing based on its potential as a biological control agent for the pathogenic bacterium Vibrio ordalii; there are no previous reports describing the isolation and characterization of a lytic phage against this bacterium. The present study represents a first step towards better understanding the roles of bacteriophages in the ecology and virulence of $V$. ordalii and contributes to the limited data sets of vibriophage and podovirus genomes. All processes, including the isolation, multiplication, count and selection of phages, and their DNA extraction/purification were performed in the Laboratorio de Microbiología Costera at the University of Antofagasta (see following section for more details). The DNA sequencing and assembly was performed at the Molecular Research Laboratory MRDNA (Shallowater, TX). The annotation and submission was performed in-house, using openly accessible informatics tools (see previous section for more details). The genome project was recorded in the NCBI database under the accession number PRJNA290661 and the genome annotation was deposited in GenBank under the accession number KT345706. A summary of the phage's features is shown in Table 1 and detailed project information is given in Table 2.

\section{Growth conditions and genomic DNA preparation}

The phage multiplication was performed applying the double-layer agar plates method [26, 27]. Vibrio ordalii cells (conc. $5 \mathrm{E}+06$ cells $/ \mathrm{mL}$ ) in the soft layer of Tryptone Soy Agar, (Oxoid, UK) were subjected to six serial dilutions (1:10-1:1000000) of phage and the lysis plaques

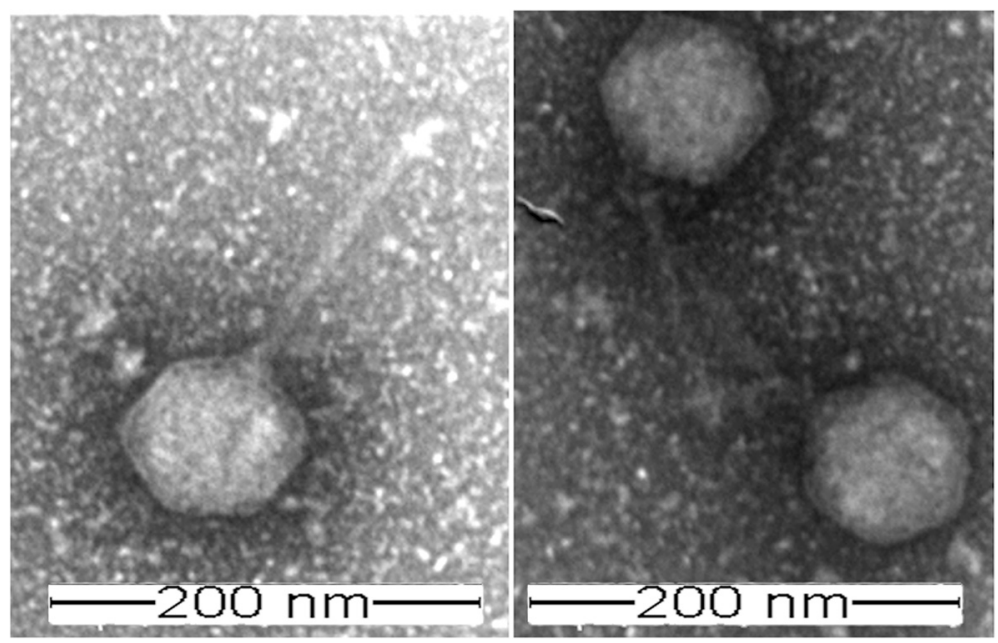

Fig. 1 Transmission electron micrograph of Vibrio phage vB_VorS-PVo5. Scale bar $=200 \mathrm{~nm}$ 


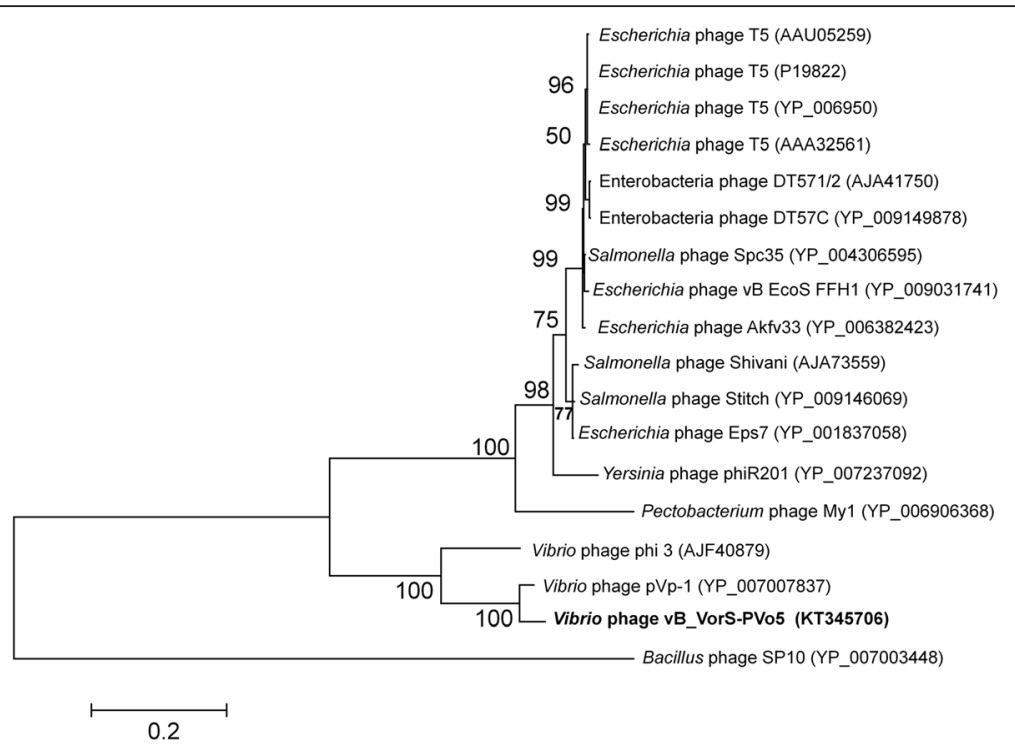

Fig. 2 Phylogenetic tree highlighting the relatedness between the Vibrio phage VB_VorS-PVo5 (shown in bold) and other viruses. The tree is based on aligned sequences of DNA polymerases over $50 \%$ similarity by BLASTP, using the Bacillus phage SP10 as the outgroup. The DNA polymerase sequence is 863 aa in length. The bootstrap consensus was set to 1000 replicates

formed were counted to determine the total number of phage. For the DNA extraction each of three plates were inoculated with 1000 PFU phage and incubated for $16 \mathrm{~h}$ at $25{ }^{\circ} \mathrm{C}$. Viral particles were re-suspended in $4 \mathrm{~mL}$ phage buffer [28] and incubated for $4 \mathrm{~h}$ with intervals of gentle shaking at $30 \mathrm{~min}$. Subsequently the supernatant was transferred into $15 \mathrm{~mL}$ falcon tubes, a $1 \mathrm{~mL}$ chloroform solution was added, gently shaken for $30 \mathrm{~s}$ and centrifuged at $5000 \mathrm{rpm}$ for $5 \mathrm{~min}$. The product was filtered through $0.22 \mu \mathrm{m}$ nitrocellulose filters (Merck-Millipore, Germany) to eliminate bigger cells. For the elimination of all external genomic content, DNase I (Thermo-Fisher, Germany) and RNase-A (Thermo-Fisher, Germany) were added at a final concentration of 5 units $\mathrm{mL}^{-1}$ each, for an incubation time of $30 \mathrm{~min}$ at $37^{\circ} \mathrm{C}$. Subsequently the flocculant PEG-80 was added at a 4:10 ratio and incubated overnight at $4{ }^{\circ} \mathrm{C}$. Viral particles were pelleted in a centrifugation step at $10000 \times \mathrm{g}$ for $1 \mathrm{~h}$ at $4{ }^{\circ} \mathrm{C}$. The supernatant was removed, the pellet dried for $\sim 5 \mathrm{~min}$ under a sterile hood, a $50 \mu \mathrm{l}$ of 10 units $\mathrm{mL}^{-1}$ Proteinase $\mathrm{K}$ (Thermo-Fisher, Germany) and phage buffer mix added, and incubated at $50{ }^{\circ} \mathrm{C}$ for $30 \mathrm{~min}$ to inactivate nucleases. The genomic DNA of the vB_VorS-PVo5 phage was extracted with a Phage DNA Isolation Kit (Norgen Biotek Corp., Canada), and evaluated and quantified with a UV-VIS spectrophotometer (BioTek Epoch, USA). In order to confirm the type of nucleic acid extracted, the product was digested separately in 1 unit/mL DNAse I and RNAse A, respectively. DNAse I, as opposed to RNAse A, degraded the extract, confirming the organism to be a DNAcontaining phage.

\section{Genome sequencing and assembly}

The genome was sequenced on an Illumina MiSeq platform at the MR-DNA Laboratory (Shallowater, TX). The library for each sample was prepared using a Nextera DNA Sample Preparation Kit (Illumina), following the manufacturer's instructions. $2 \times 130$-bp paired-end reads allowed for an estimate of 50.000 output sequences of 287 bp length with 45,367 reads remaining after the quality filtering. The assemblage of quality-filtered reads was executed for the complete genome sequence, using the pipeline by MR-DNA and resulted in an average coverage of 130 fold. A single contig of 80,578 bp corresponding to the linear genome was assembled using NGEN (DNASTAR ${ }^{\circ}$ ) by MR-DNA.

\section{Genome annotation}

The prediction of open reading frames and the comparative analysis were performed combining two methods: the PHAST server [29] and Glimmer 2.1 [30]. For the assignment of protein functions to ORFs a combination of an automatic and a manual method was used, i.e., the PHAST server and BLASTp against the NCBI nonredundant database. Only homologues with E-values $<1 \mathrm{e}-5$ were present in the annotations. The tRNA genes were searched using the tRNAscan-SE 1.21 tool [31] and TMHMM [32], and SignalP [33] were used to predict transmembrane helices and signal peptides, respectively.

\section{Genome properties}

The genome statistics are summarized in Table 3. The double-stranded and non-redundant DNA genome displayed a length of 80,578 bp with a $\mathrm{G}+\mathrm{C}$ content of 
Table 1 Classification and general features of Vibrio phage vB_VorS-PVo5

\begin{tabular}{|c|c|c|c|}
\hline MIGS ID & Property & Term & Evidence code $^{a}$ \\
\hline & Classification & Domain: viruses, dsDNA viruses, no RNA phage & TAS [39] \\
\hline & & Phylum: unassigned & \\
\hline & & Class: unassigned & \\
\hline & & Order: Caudovirales & TAS [39] \\
\hline & & Family: Siphoviridae & TAS [39] \\
\hline & & Genus: T5likevirus & TAS [39] \\
\hline & & Species: unassigned & \\
\hline & & (Type) strain: vB_VorS-PVo5 (KT345706) & \\
\hline & Particle shape & Icosahedral head with a long tail & IDA \\
\hline & Motility & non-motile & IDA \\
\hline & Sporulation & Not reported & IDA \\
\hline & Temperature range & $14-28^{\circ} \mathrm{C}$ & IDA \\
\hline & Optimum temperature & $25^{\circ} \mathrm{C}$ & IDA \\
\hline & $\mathrm{pH}$ range; Optimum & $6.5-7.5$ & IDA \\
\hline & Carbon source & Host cell & IDA \\
\hline MIGS-6 & Habitat & Oceanic, coastal & IDA \\
\hline MIGS-6.3 & Salinity & $35 \% \mathrm{NaCl}(\mathrm{w} / \mathrm{v})$ & IDA \\
\hline MIGS-22 & Oxygen requirement & Facultative aerobic & IDA \\
\hline MIGS-15 & Biotic relationship & Obligate intracellular parasite of Vibrio ordalii & IDA \\
\hline MIGS-14 & Pathogenicity & Lytic virus of Vibrio ordalii & IDA \\
\hline MIGS-4 & Geographic location & Chile/Antofagasta & IDA \\
\hline MIGS-5 & Sample collection & Jan 252014 & IDA \\
\hline MIGS-4.1 & Latitude & $23.65 \mathrm{~S}$ & IDA \\
\hline MIGS-4.2 & Longitude & $70.5 \mathrm{E}$ & IDA \\
\hline MIGS-4.4 & Altitude & $0 \mathrm{~m}$ & IDA \\
\hline
\end{tabular}

aEvidence codes - IDA: Inferred from Direct Assay; TAS: Traceable Author Statement (i.e., a direct report exists in the literature); NAS: Non-traceable Author Statement (i.e., not directly observed for the living, isolated sample, but based on a generally accepted property for the species, or anecdotal evidence). These evidence codes were obtained from the Gene Ontology project [40]

Table 2 Project information

\begin{tabular}{lll}
\hline MIGS ID & Property & Term \\
\hline MIGS 31 & Finishing quality & Complete \\
MIGS-28 & Libraries used & Illumina Myseq \\
MIGS 29 & Sequencing platforms & 130 \\
MIGS 31.2 & Fold coverage & NGEN (DNAstar) \\
MIGS 30 & Assemblers & PHAST server [29] Glimmer 2.1 [30] \\
MIGS 32 & Gene calling method & AEO54 \\
& Locus Tag & KT345706 \\
& Genbank ID & November 03 2015 \\
& GenBank Date of Release & Gp0120391 \\
& GOLD ID & PRJNA290661 \\
MIGS 13 & BIOPROJECT & Personal culture collection \\
& Source Material Identifier & Aquaculture \\
\hline
\end{tabular}


Table 3 Genome statistics

\begin{tabular}{lll}
\hline Attribute & Value & \% of the Total \\
\hline Genome size (bp) & 80,578 & 100.00 \\
DNA coding (bp) & 72,239 & 90.00 \\
DNA G + C (bp) & 32,674 & 40.55 \\
DNA scaffolds & 0 & 0.00 \\
Total genes & 103 & 100 \\
Protein coding genes & 93 & 90.29 \\
RNA genes & 10 & 9.71 \\
Pseudo genes & 0 & 0.00 \\
Genes in internal clusters & 0 & 0.00 \\
Genes with function prediction & 34 & 36.56 \\
Genes assigned to COGs & 33 & 35.49 \\
Genes with Pfam domains & 0 & 0.00 \\
Genes with signal peptides & 1 & 1.08 \\
Genes with transmembrane helices & 1 & 1.08 \\
CRISPR repeats & 1 & -
\end{tabular}

${ }^{a}$ The total is based on either the size of the genome in base pairs or the total number of protein-coding genes in the annotated genome

$40.55 \%$. A total of 10 tRNAs and 93 ORFs were identified, with no bacterial matches found. Putative functions of the identified ORFs were clustered by function [34], revealing DNA genes related to: metabolism (16), head/capsid proteins (7), phage tails (7), phage fibers and fiber assemblies (2), endolysin (1), and transcriptional regulation (1). The remaining 59 ORFs did not match any known function. The COG functional categories of identified genes are presented in Table 4, whereas the gene map is displayed in Fig. 3. The phylogenetic tree was constructed based on aligned sequences of DNA polymerases (Fig. 2). All sequences were collected from NCBI and aligned using CLUSTALW [24]; their evolutionary analysis was inferred through the neighborjoining method using MEGA6 [25].

\section{Insights from the genome sequence}

Based on its morphological characteristics, the phage vB_VorS-PVo5 was attributed to the family of the Siphoviridae. Gene comparisons confirmed the relatedness to other members in the family. 93 ORFs code for proteins homologous to known phages, 82 of which show similarity with Vibrio phage pVp-1, (GenBank accession number JQ340389), indicating a close genetic relationship between these two phages (Fig. 4). The phylogenetic tree comparing DNA-Polymerase genes confirms this similarity, forming a robust cluster supported by high bootstrap values (Fig. 3), and therefore suggests a high degree of relatedness between vB_VorS-PVo5 and other Vibrio phages. A whole genome comparison of Vibrio phage vB_VorS-PVo5, and Vibrio phage pVp-1, was performed using the Artemis Comparison Tool [35].
Table 4 Number of genes associated with general COG functional categories

\begin{tabular}{|c|c|c|c|}
\hline Code & Value & $\%$ of Total ${ }^{a}$ & Description \\
\hline J & & 0 & $\begin{array}{l}\text { Translation, ribosomal structure and } \\
\text { biogenesis }\end{array}$ \\
\hline A & & 0 & RNA processing and modification \\
\hline K & 1 & 1,08 & Transcription \\
\hline L & 13 & 13,98 & Replication, recombination and repair \\
\hline B & & 0 & Chromatin structure and dynamics \\
\hline D & & 0 & $\begin{array}{l}\text { Cell cycle control, Cell division, } \\
\text { chromosome partitioning }\end{array}$ \\
\hline V & & 0 & Defense mechanisms \\
\hline $\mathrm{T}$ & & 0 & Signal transduction mechanisms \\
\hline M & & 0 & Cell wall/membrane biogenesis \\
\hline N & & 0 & Cell motility \\
\hline U & & 0 & Intracellular trafficking and secretion \\
\hline $\mathrm{O}$ & & 0 & $\begin{array}{l}\text { Posttranslational modification, protein } \\
\text { turnover, chaperones }\end{array}$ \\
\hline C & & 0 & Energy production and conversion \\
\hline G & & 0 & Carbohydrate transport and metabolism \\
\hline $\mathrm{E}$ & & 0 & Amino acid transport and metabolism \\
\hline $\mathrm{F}$ & 1 & 1,08 & Nucleotide transport and metabolism \\
\hline $\mathrm{H}$ & & 0 & Coenzyme transport and metabolism \\
\hline I & & 0 & Lipid transport and metabolism \\
\hline$P$ & & 0 & Inorganic ion transport and metabolism \\
\hline Q & & 0 & $\begin{array}{l}\text { Secondary metabolites biosynthesis, } \\
\text { transport and catabolism }\end{array}$ \\
\hline $\mathrm{R}$ & & 0 & General function prediction only \\
\hline$S$ & 59 & 63,44 & Function unknown \\
\hline- & 1 & 1,08 & Not in COGs \\
\hline$x$ & 18 & 19,35 & Mobilome: Prophage, Transposons \\
\hline
\end{tabular}

${ }^{a}$ The total is based on the total number of protein coding genes in the genome

Genomes were aligned by WebACT, using the default tblastx settings (E-value: 10e-4, genetic code: eubacterial). The ACT display was set to show homologous regions with BLAST scores of $>40$, and a sequence similarity of $>25 \%$.

Phage reproduction may involve either a lytic or a lysogenic cycle with some viruses being capable of performing both. In the lysogenic cycle the viral genome will integrate with host DNA and replicate along with it, whereas the lytic phage will destroy the host cell immediately after the replication, breaking bacterial cells open and allowing the phage progeny to find new hosts to infect. Due to quickly destroying the bacterial cells, lytic phages are more suitable for phage therapy, and the genes coding for the production of endolysin are the key evidence for the phage's lytic characteristics [36, 37]. A BLASTP comparison of the endolysin sequences displayed 


Fig. 3 Gene map of the Vibrio phage $\mathrm{VB}$ VorS-PVO5

a similarity of $94 \%$ and $46 \%$ with the Vibrio phage pVp-1 and Vibrio phage Phi-3, respectively. Furthermore, the enzyme destroys bacterial cell walls and has therefore been discussed for its use as an anti-infective to control pathogens [36, 38].

The specificity of the phage vB_VorS-PVo5 has been tested on Vibrio anguillarum, the most closely related species to Vibrio ordalii [8] and another Chilean strain of Vibrio ordalii, isolated from scallops, and whereas vB_VorS-PVo5 infected both Vibrio ordalii species it did not lyse Vibrio anguillarum. More research has to be done in order to test the effect of vB_VorS-PVo5 phage and its endolysin as a therapy, however, the fact that only Vibrio ordalii strains were lysed indicates that vB_VorS-PVo5 might be highly species specific and may therefore prove to be a very promising candidate for phage therapy against Vibrio ordalii.

\section{Conclusions}

Here we report for the first time on the isolation and genome sequencing of $\mathrm{vB}$ _VorS-PVo5 a novel phage that belongs to the family of the Siphoviridae and is

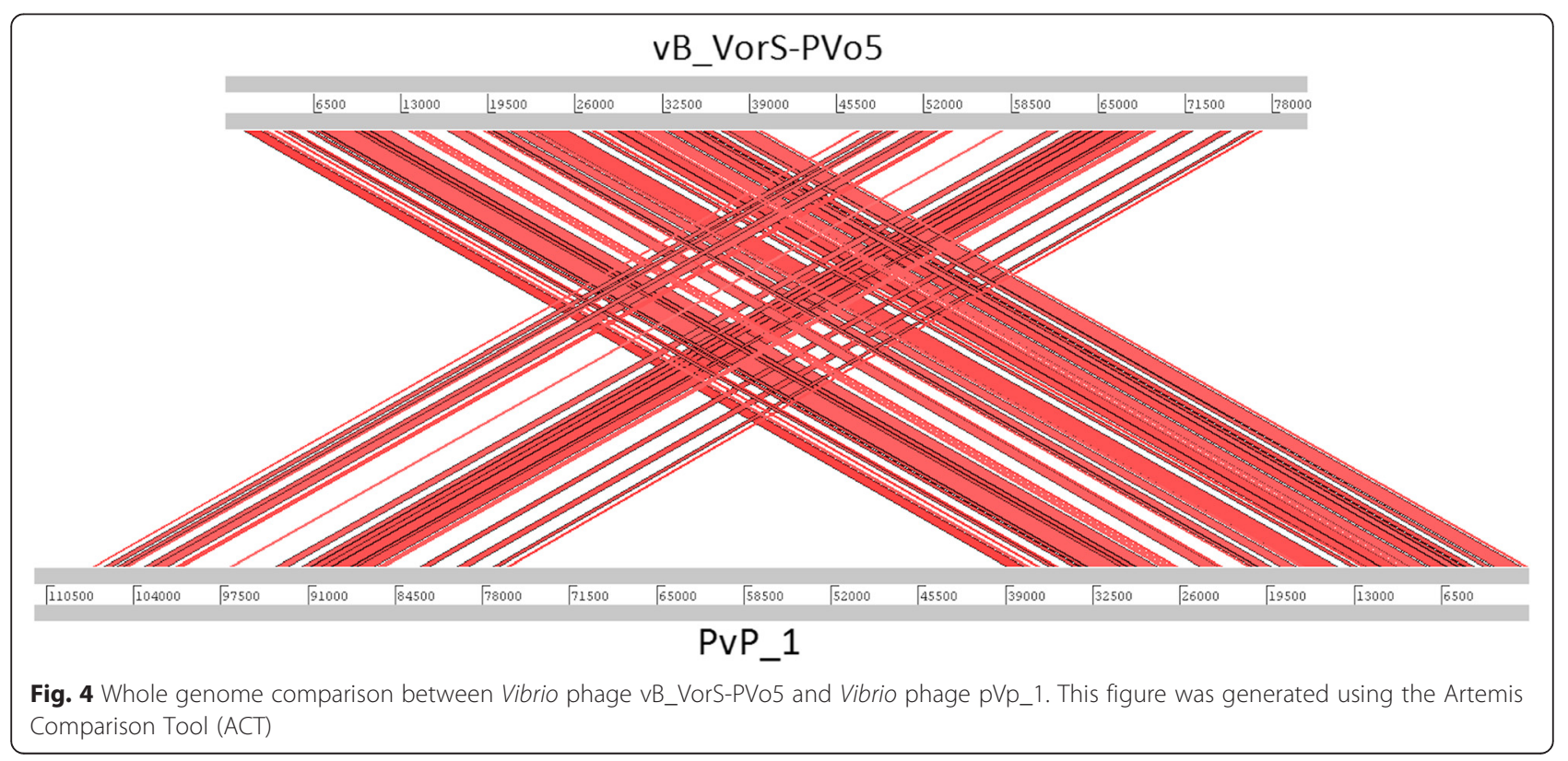


capable of lysing the pathogen Vibrio ordalii ATCC 33509. The lytic character of the phage, together with the first indication of its specificity for Vibrio ordalii strains indicates the potential for its future use in aquaculture applications, controlling the pathogen either by using the phage or its endolysin.

\section{Acknowledgements}

We would like to thank Roberto Bastías for providing microbial strains. This work was funded by the following grants: Project CORFO FIC-R 13IDL2-18530 to RA, and Beca de Doctorado CONICYT FIC-R N²1092008 and Beca de Apoyo de Tesis, Programa de Doctorado en Ciencias Aplicadas, Mención Sistemas Marinos Costeros 2013-2014 to AE.

\section{Authors' contributions}

AE conceived the study, participated in all samplings, performed sample preparation, conducted all molecular genetic studies and drafted the manuscript. PM participated in samplings, culture maintenance, sample preparation and molecular genetic studies. CS participated in samplings, culture maintenance, sample preparation and molecular genetic studies. $\mathrm{JC}$ assisted in drafting the manuscript. RA participated in the experimental design, coordinated the project and assisted in drafting the manuscript. All authors have read and approved the final manuscript.

\section{Competing interests}

The authors declare that they have no competing interests.

\section{Author details}

'Programa de Doctorado en Ciencias Aplicadas, Mención Sistemas Marinos Costeros, Facultad de Ciencias del Mar y Recursos Marinos, Universidad de Antofagasta, Angamos 601, Antofagasta, Chile. '2Laboratorio de Microbiología Costera, Facultad de Ciencias del Mar y Recursos Biológicos, Universidad de Antofagasta, Angamos 601, Antofagasta, Chile. Instituto de Ciencias Naturales Alexander von Humboldt, Facultad de Ciencias del Mar y Recursos Biológicos, Universidad de Antofagasta, Angamos 601, Antofagasta, Chile.

Received: 25 October 2015 Accepted: 3 May 2016

Published online: 04 July 2016

\section{References}

1. Aravena G, Broitman B, Stenseth NC. Twelve years of change in coastal upwelling along the Central-Northern Coast of Chile: spatially heterogeneous responses to climatic variability. PLoS ONE. 2014;9(2):e90276.

2. Taylor MH, Wolff M. Trophic modeling of Eastern Boundary Current Systems: a review and prospectus for solving the "Peruvian Puzzle". Rev Peru Biol. 2007;14:87-100.

3. Neale P, Sobrino C, Segovia M, Mercado J, Leon P, Cortés M, Tuite P, Picazo A, Salles S, Cabrerizo M, Prasil O, Montecino V, Reul A, and Fuentes-Lema A. Effect of $\mathrm{CO} 2$, nutrients and light on coastal plankton. I. Abiotic conditions and biological responses. Aquat Biol. 2014;22:25-41.

4. Leyton Y, Riquelme C. Vibrios en los sistemas marinos costeros. Rev Biol Mar Oceanogr. 2008:43:441-56.

5. Paillard C, Le Roux F, Borrego J. Bacterial disease in marine bivalves, a review of recent studies: trends and evolution. Aquat Living Resour. 2004;17(04):477-98.

6. Bohle H, Kjetil F, Bustos P, Riofrío A, Peters C. Fenotipo atípico de Vibrio ordalii, bacteria altamente patogénica aislada desde salmón del Atlántico cultivado en las costas marinas del sur de Chile. Arch Med Vet. 2007;39:43-52.

7. Muller M, llardi P, Avendaño-Herrera R. Eficacia de un desinfectante sobre Vibrio ordalii, Vibrio anguillarum, Francisella sp. y Virus de la necrosis Pancreática infecciosa (IPNV), patógenos de salmón del atlántico (Salmo salar) cultivado en Chile. Arch Med Vet. 2011;43(1):73-8.

8. Schiewe M, T. Trust, Crosa J. Vibrio ordalii sp. nov.: a causative agent of vibriosis in fish. Curr Microbiol. 1981;6(6):343-8

9. Silva-Rubio A, Acevedo C, Magariños B, Jaureguiberry B, Toranzo A, Avendaño-Herrera R. Antigenic and molecular characterization of Vibrio ordalii strains isolated from Atlantic salmon Salmo salar in Chile. Dis Aquat Org. 2008;79(1):27-35.

10. Alderman DJ, Hastings TS. Antibiotic use in aquaculture: development of antibiotic resistance - potential for consumer health risks. Int J Food Sci Technol. 1998;33(2):139-55.
11. Romero J, Feijoo CG, Navarrete P. Antibiotics in aquaculture - use, abuse and alternatives. In: Gianmarco Silva D, Silva RJ, Carvalho E, editors. Health and environment in aquaculture. Vol. 1. Rijeka: InTech 40; 2012.

12. Cabello FC. Antibióticos y acuicultura en Chile: consecuencias para la salud humana y animal. Rev Med Chil. 2004;132:1001-6.

13. Boyd CE, Massaut L. Risks associated with the use of chemicals in pond aquaculture. Aquac Eng. 1999;20(2):113-32.

14. Cabello FC. Heavy use of prophylactic antibiotics in aquaculture: a growing problem for human and animal health and for the environment. Environ Microbiol. 2006;8(7):1137-44.

15. Palmer AC, Angelino E, Kishony R. Chemical decay of an antibiotic inverts selection for resistance. Nat Chem Biol. 2009;6(2):105-7.

16. Foster PL. Stress-induced mutagenesis in bacteria. Crit Rev Biochem Mol Biol. 2007;42(5):373-97.

17. Done HY, Halden RU. Reconnaissance of 47 antibiotics and associated microbial risks in seafood sold in the United States. J Hazard Mater. 2015;282:10-7.

18. Kutter EM, Kuhl SJ, Abedon ST. Re-establishing a place for phage therapy in western medicine. Future Microbiol. 2015;10(5):685-8.

19. Sarhan WA, Azzazy HM. Phage approved in food, why not as a therapeutic? Expert Rev Anti-Infect Ther. 2015;13(1):91-101.

20. Verbeken G, Huys I, Pirnay J-P, Jennes S, Chanishvili N, Scheres J, Chanishvili N, Scheres J, Górski A, De Vos D, and Ceulemans C. Taking bacteriophage therapy seriously: a moral argument. BioMed Res Int. 2014;2014:8.

21. Keen EC, Adhya SL. Review of phage therapy: current research and applications. Clin Infect Dis. 2015. doi:10.1093/cid/civ257.

22. Chen F, Wang K, Huang S, Cai H, Zhao M, Jiao N, and Wommack KE. Diverse and dynamic populations of cyanobacterial podoviruses in the Chesapeake Bay unveiled through DNA polymerase gene sequences. Environ Microbiol. 2009;11(11):2884-92

23. Kim JH, Jun JW, Choresca CH, Shin SP, Han JE, Park SC. Complete genome sequence of a novel marine siphovirus, pVp-1, infecting vibrio parahaemolyticus. J Virol. 2012;86(12):7013-4.

24. Thompson JD, Higgins DG, Gibson TJ. CLUSTAL W: improving the sensitivity of progressive multiple sequence alignment through sequence weighting, position-specific gap penalties and weight matrix choice. Nucleic Acids Res. 1994:22(22):4673-80.

25. Tamura K, Stecher G, Peterson D, Filipski A, Kumar S. MEGA6: molecular evolutionary genetics analysis version 6.0. Mol Biol Evol. 2013;30(12):2725-9.

26. Gratia A. Numerical relations between lysogenic bacteria and particles of bacteriophage. Ann Inst Pasteur. 1936;57(652-676):72.

27. Suttle CA, Chen F. Mechanisms and rates of decay of marine viruses in seawater. Appl Environ Microbiol. 1992:58(11):3721-9.

28. Boyle JM, Symonds N. Radiation-sensitive mutants of T4D I. T4y: A new radiation-sensitive mutant: Effect of the mutation on radiation survival, growth and recombination. Mutat Res Fundam Mol Mech Mutagen. 1969;8(3):431-9.

29. Zhou Y, Liang Y, Lynch KH, Dennis JJ, Wishart DS. PHAST: A Fast Phage Search Tool. Nucleic Acids Res. 2011. 37. Effantin, G., P. Boulanger, E. Neumann, L. Letellier, and J.F. Conway, Bacteriophage T5 structure reveals similarities with HK97 and T4 suggesting evolutionary relationships. Journal of Molecular Biology, 2006. 361(5): p. 993-1002.

30. Delcher AL, Harmon D, Kasif S, White O, Salzberg SL. Improved microbial gene identification with GLIMMER. Nucleic Acids Res. 1999;27(23):4636-41.

31. Schattner P, Brooks AN, Lowe TM. The tRNAscan-SE, snoscan and snoGPS web servers for the detection of tRNAs and snoRNAs. Nucleic Acids Res. 2005;33 suppl 2:W686-9.

32. Krogh A, Larsson B, von Heijne G, Sonnhammer ELL. Predicting transmembrane protein topology with a hidden Markov model: application to complete genomes. J Mol Biol. 2001;305(3):567-80.

33. Petersen TN, Brunak S, von Heijne G, Nielsen H. SignalP 4.0: discriminating signal peptides from transmembrane regions. Nat Methods. 2011:8:785-6.

34. Lima-Mendez G, Toussaint A, Leplae R. Analysis of the phage sequence space: the benefit of structured information. Virology. 2007;365(2):241-9.

35. Carver TJ, Rutherford KM, Berriman M, Rajandream M-A, Barrell BG, Parkhill J. ACT: the Artemis Comparison Tool. Bioinformatics (Oxford, England). 2005; 21(16):3422-3.

36. Yang $\mathrm{H}, \mathrm{Yu}$ J, Wei $\mathrm{H}$. Engineered bacteriophage lysins as novel antiinfectives. Front Microbiol. 2014;5:542. 
37. Wang I-N. Lysis timing and bacteriophage fitness. Genetics. 2006;172(1):17-26.38.

38. Fischetti VA. Bacteriophage endolysins: a novel anti-infective to control Gram-positive pathogens. Int J Med Microbiol. 2010;300(6):357-62.

39. King AM, Adams MJ, Carstens EB, Lefkowitz EJ. Virus taxonomy: Ninth report of the International Committee on Taxonomy of Viruses. San Diego: Elsevier; 2012.

40. shburner M, Ball CA, Blake JA, Botstein D, Butler H, Cherry JM, Davis AP, Dolinski K, Dwight SS, Eppig JT, Harris MA, Hill DP, Issel-Tarver L, Kasarskis A, Lewis S, Matese JC, Richardson JE, Ringwald M, Rubin GM, and Sherlock G. Gene ontology: tool for the unification of biology. Nat Genet. 2000;25(1):25-9.

Submit your next manuscript to BioMed Central and we will help you at every step:

- We accept pre-submission inquiries

- Our selector tool helps you to find the most relevant journal

- We provide round the clock customer support

- Convenient online submission

- Thorough peer review

- Inclusion in PubMed and all major indexing services

- Maximum visibility for your research

Submit your manuscript at www.biomedcentral.com/submit
() BioMed Central 